\title{
A Decade of DTI in Traumatic Brain Injury: 10 Years and 100 Articles Later
}

M.B. Hulkower, D.B. Poliak, S.B. Rosenbaum, M.E. Zimmerman, and M.L. Lipton

Q- $=$

\begin{abstract}
SUMMARY: The past decade has seen an increase in the number of articles reporting the use of DTI to detect brain abnormalities in patients with traumatic brain injury. DTI is well-suited to the interrogation of white matter microstructure, the most important location of pathology in TBI. Additionally, studies in animal models have demonstrated the correlation of DTI findings and TBI pathology. One hundred articles met the inclusion criteria for this quantitative literature review. Despite significant variability in sample characteristics, technical aspects of imaging, and analysis approaches, the consensus is that DTI effectively differentiates patients with TBI and controls, regardless of the severity and timeframe following injury. Furthermore, many have established a relationship between DTI measures and TBI outcomes. However, the heterogeneity of specific outcome measures used limits interpretation of the literature. Similarly, few longitudinal studies have been performed, limiting inferences regarding the long-term predictive utility of DTI. Larger longitudinal studies, using standardized imaging, analysis approaches, and outcome measures will help realize the promise of DTI as a prognostic tool in the care of patients with TBI.
\end{abstract}

ABBREVIATIONS: FA = fractional anisotropy; GCS = Glasgow Coma Scale; $M D=$ mean diffusivity; TAI = traumatic axonal injury; TBI = traumatic brain injury; TBSS $=$ tract-based spatial statistics

$\mathrm{T}$ he clinical pathology underlying TBI-related impairment is traumatic axonal injury. ${ }^{1}$ TAI, referred to as diffuse axonal injury when damage is extensive, is a microscopic injury that occurs even in the absence of frank tissue disruption. Therefore, patients may experience significant impairment despite the absence of abnormal findings on conventional CT and MR imaging. Moreover, focal imaging abnormalities that can be detected by using CT and MR imaging are poor predictors of outcome. ${ }^{1} \mathrm{Di}$ agnostic tests that can discriminate significant TAI are needed to effectively allocate patients to follow-up and treatment, to accurately assess injury severity and safety in sports and military settings, and to guide clinical trials of novel therapeutic agents. DTI is a relatively new MR imaging technique that measures the directional coherence of water diffusion in vivo. Because of the highly uniform collinear structure of normal white matter, DTI is uniquely able to probe its microscopic structure and is, therefore,

From the Gruss Magnetic Resonance Research Center (M.L.L.) and Saul R. Korey Department of Neurology (M.E.Z.), Albert Einstein College of Medicine (M.B.H., D.B.P., S.B.R.), Bronx, New York.

Please address correspondence to Michael Lipton, MD, PhD, The Gruss Magnetic Resonance Research Center, Albert Einstein College of Medicine, 1300 Morris Park Ave, Bronx, NY 10461; e-mail: michael.lipton@einstein.yu.edu

- Indicates open access to non-subscribers at www.ajnr.org

= Indicates article with supplemental on-line tables

http://dx.doi.org/10.3174/ajnr.A3395 particularly well-suited for the assessment of TAI. Although gross abnormalities can be identified in some cases of TAI by using CT and conventional MR imaging, DTI can both qualitatively and quantitatively (Fig 1) demonstrate pathology not detected by other modalities and is, therefore, an important tool not only in the research setting but in the clinical setting as well.

Most studies of TBI report fractional anisotropy, a summary measure derived from DTI, which describes the directional coherence (anisotropy) of water diffusion within tissue. However, mean diffusivity, axial diffusivity, and radial diffusivity may more specifically describe the direction and magnitude of tissue water diffusion. Animal studies have shown a direct correspondence between even very subtle TAI pathology and decreases in white matter anisotropy that can be imaged in vivo by using DTI (eg, Mac Donald et $\mathrm{al}^{2}$ ). Numerous clinical studies have assessed TBI by using DTI. Since the earliest research article reporting DTI applied to TBI was published in $2002,{ }^{3}$ there has been an overall exponential increase in the number of articles published on this topic (Fig 2).

The purpose of this review was to systematically summarize and detail the landscape of DTI applied to the study of TBI and to highlight both the salient conclusions to be drawn from this large literature and its limitations, which can serve as important considerations for future research. We summarize a number of different aspects of the articles, including the demo- 

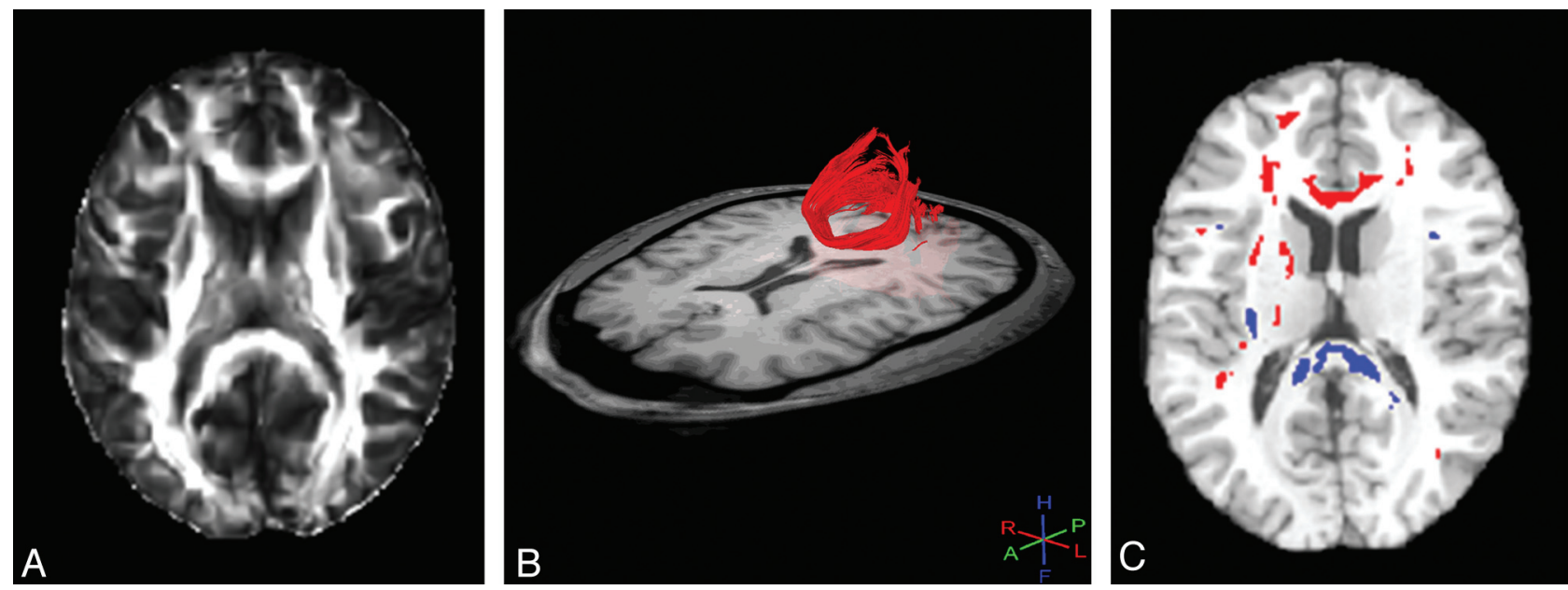

FIG 1. FA image $(A)$ reveals no abnormality in a patient with TBI. Tractography $(B)$ can be used to delineate a region of interest for analysis. In this case, the forceps major (red) appears normal, but quantitative analysis of FA within this tract showed lower FA in the TBI group compared with controls. Whole-brain voxelwise analysis (C) reveals areas of low (blue) and high (red) FA. Low FA, consistent with TAI, is present within the forceps major at the splenium of the corpus callosum, as well as elsewhere.

\section{Rate of Publication: Studies of DTI in TBI}

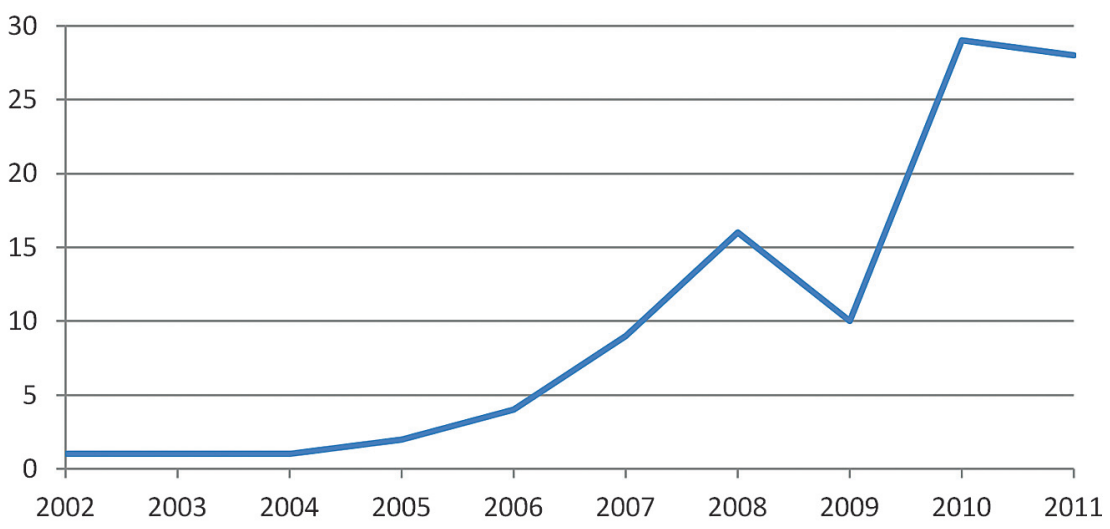

of diseases other than TBI (eg, spinal cord injury, brain tumors) $(n=57)$; case reports $(n=37)$, reviews $(n=48)$, editorials $(n=3)$, posters $(n=1)$, or abstracts $(n=1)$; and use of diffusion-weighted imaging or other MR imaging measures, but not DTI $(n=8)$.

\section{SUBJECTS WITH TBI}

The population studied or substrate of injury is perhaps as important as the TBI itself in determining the nature and extent of consequent pathology. ${ }^{103}$ An important consideration in the study of TBI is thus the choice of the study sample and the feasibility of attaining a homogeneous cohort. A total of 2337 subjects was studied across all 100 articles. The average graphics of TBI subjects and controls, the timing and severity of TBI, technical factors related to image acquisition and analysis, the nature and location of abnormalities, and findings relating DTI to outcome measures. We also note that a valid meta-analysis of this literature is not feasible due to the great diversity in study design and measurement approaches used across the articles.

A structured search was performed by using PubMed to include all relevant articles through 2011. The search used the following key word combinations: "diffusion tensor imaging and traumatic brain injury," "DTI and TBI," and "DTI and concussion." The total results included 391 articles with 293 unique articles. We further examined the references cited by these articles to identify additional relevant articles. After we eliminated articles on the basis of our exclusion criteria (below), 100 articles $^{3-102}$ remained and were systematically analyzed and included in this review. Exclusion criteria included the following: language other than English $(n=7)$; animal or in vitro studies $(n=30)$; studies number of patients per study was 23 (range, 5-83 subjects). Our review identified several articles that described patient samples with extremely similar or identical demographic characteristics (eg, McCauley et $\mathrm{al}^{55}$ and Wilde et $\mathrm{al},{ }^{87,88,90}$ ) but reported either different abnormal brain regions or different analyses of DTI in relation to outcome. Thus, some of the subjects may have been reported in multiple studies published by the same group of researchers. Our best estimate is that the number of subjects reported in multiple studies may be up to 140 individuals. All except 8 articles reported sex breakdown; $65 \%$ of reported subjects were male. ${ }^{6,30,41,46,52,77,81,83}$

Most commonly, abnormalities on DTI are defined on the basis of comparison with a control group because universal thresholds for abnormality have not yet been established. All studies, except 5, compared subjects with TBI with a control group. ${ }^{17,18,24,83,85}$ Three of these exceptions used a longitudinal within-subjects design. ${ }^{24,83,85}$ In all except 7 studies, control subjects were healthy individuals. ${ }^{28,43,49,53,55,81,90}$ In 3 of the studies 


\section{Severity and Chronicity}

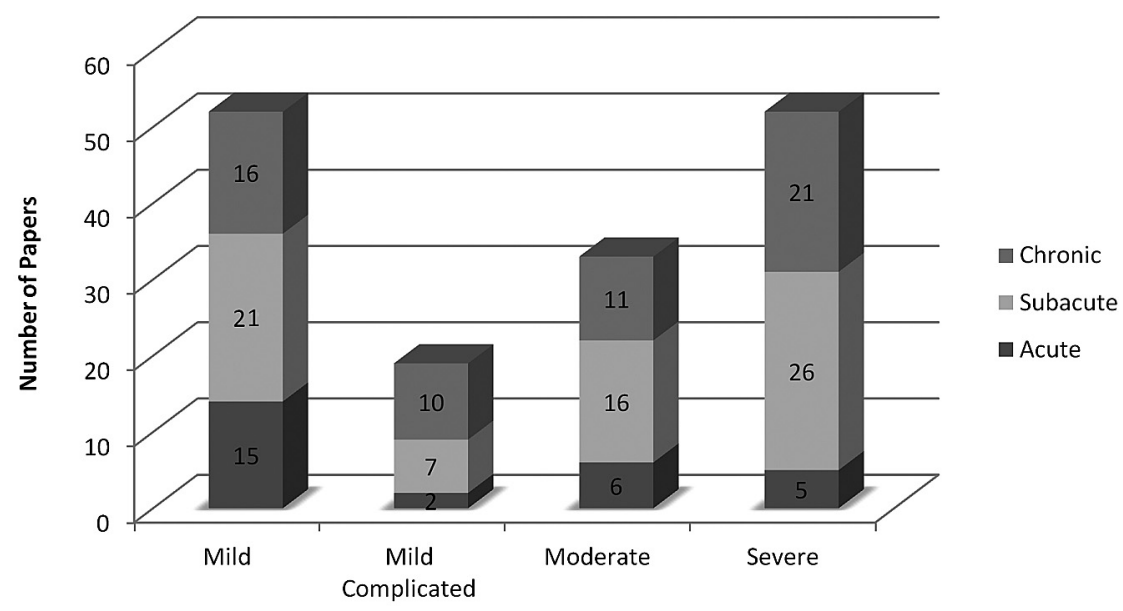

FIG 3. The number of articles that studied patients at each timeframe and level of injury severity. Articles were only included if there was sufficient information to determine both the severity and the chronicity of individual patient injuries. Articles may be included multiple times if they studied subjects with multiple severities and/or multiple chronicities. A fully referenced version of this figure is available in On-line Table I.

that did not use healthy individuals as control subjects, 2 groups of subjects with TBI were compared (eg, with and without major depressive disorders ${ }^{53}$ ). In 3 pediatric studies, the control groups were children who were hospitalized for orthopedic injury but had no evidence of head injury. ${ }^{43,55,90}$ The average number of control subjects per study was 18 (range, 6-47). Several articles, in addition to comparing patients with TBI with controls by using DTI, looked at subgroups of patients with TBI, including patients with TBI with major depressive disorders, ${ }^{53,99}$ with raised intracranial pressures, ${ }^{81}$ with spinal cord injuries, ${ }^{86}$ or veterans with blast injuries. ${ }^{41,49,53,79,99}$ An important consideration in the selection of control subjects, particularly when studying symptom endorsement, is that comparison of subjects with TBI with healthy controls fails to eliminate the potential confound of morbidity due to the experience of trauma itself, rather than adverse outcomes specifically due to physical injury to the brain. At the same time, it is unclear whether a purportedly non-head-injured patient sustained neurologic trauma during the course of an injury, even if not reported by the patient or witnesses of the event or detected by clinical assessment of the patient.

The ages of subjects studied across all articles ranged from 2 through 70 years, with each individual article reporting on a more limited age range. Children were studied exclusively in 29 of 72 articles. ${ }^{4,5,11-14,17,19-22,25,27,42,43,55,66,75,81,87-94,96,98}$ The total number of children studied across all 29 articles was 564 . In addition, 20 studies included adults and children (younger than 19 years). ${ }^{8-11,26,31,49,50,56,59,60,62,64,65,67,70,83,84,97,99}$ However, varied age thresholds were used to define the pediatric population. Most studies defined the pediatric population as individuals younger than 17 years of age, but other studies included children up to 18 years, ${ }^{4,5,92} 19$ years, ${ }^{75} 20$ years, ${ }^{12-14}$ or 22.5 years of age. ${ }^{81}$ White matter changes associated with normal development might confound detection of white matter injury; moreover, because developmental changes can occur at different rates even in children of the same chronologic age, use of DTI in the pediatric population is a challenging undertaking.

Many studies reported the mechanism of injury of patients with TBI such as motor vehicle collisions, falls, and assaults. However, because patients with different mechanisms were almost always consolidated into a single patient group, it is impossible to draw conclusions about imaging findings as they relate to different mechanisms of injury.

Because age, sex, anthropometrics, and injury mechanism can greatly influence outcomes, it is important that these issues be considered in study design and interpretation of results. Two major issues therefore emerge in consideration of the demographics of a TBI population. First, it is important to frame the comparison of results from multiple studies in the context of demographic differences between the studied samples. Second, within a single study, a group analysis involving a demographically diverse sample might mask important findings unique to a particular demographic subset or lead to spurious group differences.

\section{SEVERITY, CHRONICITY, AND STUDY DESIGN}

Traumatic injury to the brain can result in a spectrum of injuries. There is a lack of consensus regarding whether they represent subsets of a single-entity or distinct pathologic processes. We found a wide variation in the injury severity studied, ranging from mild, in which there is a complete absence of abnormalities on conventional imaging, to severe, in which subjects remain in a vegetative state. While some studies were restricted to patients of a specific injury severity, many studies included patients of varying severities. For studies reporting the GCS, we defined severity as mild (GCS, 13-15), moderate (GCS, 9-12), or severe (GCS, 3-8). For articles that did not report the GCS but characterized severity as mild, moderate, or severe, we accepted the authors' report of severity. Four studies did not report injury severity. ${ }^{26,33,53,60}$ Several articles distinguished between mild-complicated and mild-uncomplicated TBI. While both groups of patients exhibited GCS in the mild range ( $>13$ ), patients with mildcomplicated TBI had findings of TBI on conventional imaging (CT and structural MR imaging), whereas patients with "true" mild or mild-uncomplicated TBI did not. Some articles specifically reported a distinction between patients with mild-complicated and mild-uncomplicated TBI. Many articles, though not making the mild-complicated versus mild-uncomplicated distinction, specifically noted that subjects with mild TBI lacked significant findings on conventional imaging. However, other articles simply reported "mild" severity without mentioning the results of conventional brain imaging. There has been an increasing trend toward differentiating mild-complicated versus milduncomplicated injury beginning in 2009. The earlier literature did not make the distinction, referring to the injury as "mild" without 


\section{Longitudinal Studies}

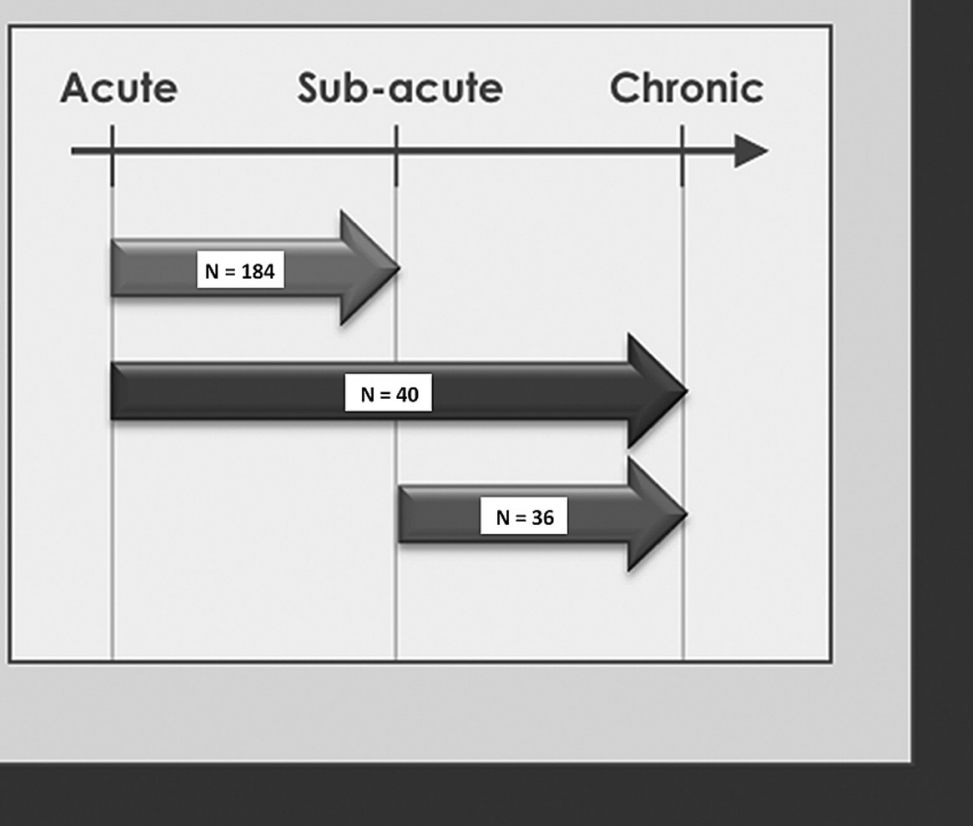

FIG 4. Thirteen studies used a longitudinal design. Numbers represent patients from all studies imaged at 2 time points. Nine studies assessed patients at both acute and subacute time points. ${ }^{3,29,39,47,54,56,67,83,85}$ One study assessed patients at both acute and chronic time points. ${ }^{102}$ Two studies assessed patients at both subacute and chronic time points. ${ }^{24,93}$ One study $(n=47)$ assessed patients twice during the subacute period and, therefore, was omitted from the figure. $^{49}$

qualification. This likely represents an increasing awareness of the distinction between mild-complicated and mild-uncomplicated, because studies have demonstrated that the clinical features of mild-complicated injuries often bear closer resemblance to those of moderate TBI than they do to mild-uncomplicated TBI. ${ }^{104}$ Finally, some articles did not differentiate moderate and severe TBI but, rather, grouped all such patients into 1 category.

Similar to severity, chronicity (Fig 3 ) is an important factor in the development of the study design. Primary injury and secondary injury play different roles in the evolution of pathology as a function of time postinjury. Similarly, microstructural pathology, as detected with DTI, may change with time. We found a wide variation among studies as to the timing of DTI relative to TBI, ranging from days to years. To systematically assess the timing of DTI after injury, which we termed "injury chronicity," we divided subjects into 3 generally accepted categories. Acute injury included patients imaged within 2 weeks of TBI, while chronic injury included patients imaged at least 1 year following injury. The subacute period included imaging performed between 2 weeks and 1 year after TBI. All articles reported the timing of DTI after TBI.

Figure 3 details the total number of articles reporting DTI for the study of TBI within a given timeframe and of a particular injury severity and includes articles that report mixed severities, mixed chronicities, or both. Most subjects were studied in the subacute setting (Fig 3), most commonly having severe TBI followed by mild TBI. This trend might be attributable to the greater ease of subject enrollment during the subacute period, rather than to the clinical importance of this timeframe for the study. Patients are more easily enrolled from rehabilitation centers, where they were actively seeking treatment for their injuries. In the acute setting, patients with mild TBI might not even seek medical treatment and patients with severe TBI were too involved in urgent treatment to participate in a research study. Unless identified retrospectively, patients are easily lost to follow-up at chronic time points. When categorized according to severity, independent of chronicity, the total number of patients with moderate TBI represented approximately half the total number of patients with mild TBI and half the total number of patients with severe TBI (Fig 3). Although the minority of patients studied across all severities was examined in the acute setting, use of DTI as a prognostic tool is dependent on the identification of early biomarkers; therefore, the acute setting is an important area for future research, as are longitudinal studies examining patients at both the acute and chronic time points.

Most articles used cross-sectional designs. Eighteen articles reported multiple groups of subjects who were imaged at various times postinjury, ${ }^{3,5,8,24,25,31,32,34,39,54,56,65,68,72,76,85,93,102}$ whereas 79 articles reported a single homogeneous group of patients all within a 1 -injury chronicity timeframe. Only 13 articles, reporting a total of 283 patients, used longitudinal designs and imaged the same group of patients at 2 different time points (Fig 4). ${ }^{3,24,29,39,47,49,54,56,67,83,85,93,102}$ One additional article reported imaging of the same group of patients acutely and again at least 1 year following injury but used a cross-sectional analysis at each of these time points and did not report change with time within subjects. ${ }^{85}$

A prospective study of patients with TBI is difficult; attrition during follow-up is a significant challenge. Of those studies that used a longitudinal design, only 8 reported attrition. The average attrition rate across these studies was 0.32 (range, 0.110.60). ${ }^{3,24,29,49,54,56,83,85}$ Despite the challenge of attrition, longitudinal studies are integral to the understanding the natural history of TBI and for early prognostication.

\section{DATA ACQUISITION PARAMETERS}

Important considerations in the use of DTI are the strength of the magnetic field, the number of diffusion-sensitizing gradient magnetic field directions, and the choice of $b$-values. Use of greater magnetic field strengths has advantages and disadvantages; greater signal-to-noise ratio, improved spatial resolution, and

AJNR Am J Neuroradiol 34:2064-74 Nov 2013 www.ajnr.org 
faster scanning times commonly achieved at stronger magnetic field strengths come at the cost of increased magnetic field inhomogeneity. In the setting of clinical DTI, most facilities will choose a b-value between 750 and 1000, whereas b-values of up to 3000 are used experimentally. ${ }^{105,106}$ Increasing the b-value increases the sensitivity to diffusion, but with a decrease in signalto-noise ratio. Encoding the direction of diffusion requires a minimum of 6 diffusion-sensitizing directions; greater resolution can be achieved by introducing additional directions, but this adds time to image acquisition. We summarize the acquisition parameters used in DTI studies of TBI as follows.

Nearly equal numbers of articles reported performing DTI

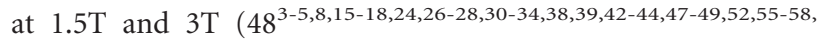
$66-68,70,72,73,76,77,79,80,82,87,88,91,93,97,101,102$ and $52,6,7,9-14,19-23,25$, 29,35-37,40,41,45,46,50,51,53,54,59-65,69,71,74,75,78,81,83-86,89,90,92,94-96,98-100

respectively). The number of diffusion-sensitizing directions used ranged from 6 to 64, with an average of 27 but a mode of 12. In 2011 alone, the mode increased to 64 . The b-value was reported in all except 6 articles. ${ }^{24,53,74,96,99,100}$ All articles except 7 used a single bvalue in addition to zero. ${ }^{25,59-63,81}$ The 7 articles using multiple b-values each used 5 unique b-values. The average b-value was 947, the range of b-values was 300-1590, and the median and the mode were 1000 .

Spatial resolution is an additional important consideration. Assessments of anisotropy, which are central to studies of TBI, measure the aggregate range of diffusivities across the tissues composing the voxel. Partial volume effects that may spuriously reduce anisotropy will be more likely when larger voxel volumes are examined. Voxel sizes varied among the articles we reviewed, with an average voxel size of $11.3 \mathrm{~mL}^{3}$ (range, $1.83-31.25 \mathrm{~mL}^{3}$ ). Average section thickness was $3.08 \mathrm{~mm}$ (range, $1.72-6 \mathrm{~mm}$ ).

\section{DATA ANALYSIS METHODS}

DTI can be used to study brain structure either on a regional or a whole-brain level. Regional analyses include both those in which an a priori region of interest is chosen for study and tractography, in which an a priori region of interest is used to define a white matter tract for study. In both approaches, average diffusion values such as FA are extracted from voxels within the ROIs or tracts for subsequent analysis. Whole-brain analyses include voxelwise analyses, tract-based spatial statistics, a specialized type of voxelwise analysis, and histogram analyses of all brain or all white matter voxels.

In our review of the literature, we found that among those studies using regional analyses, 60 studies $^{3-6,10,12-19,22-28,30,33-35 \text {, }}$ $37-41,43,46,48,49,51,52,54,55,57-66,76,81,82,86,92,93,95-100,102$ used a region-of-interest approach and 30 studies $9,11,17-19,21,31,41,42,46$, $47,50,55,58,63,67,69,72,73,75,77,79,80,83,84,87,88,91,95,101$ used tractography. Fewer studies used whole-brain analyses, of which 17 studies $^{7,11,20,21,29,44,45,50,53,56,58,71,74,80,95,98,101}$ were based on a voxelwise approach, 7 studies ${ }^{14,32,36,68,78,86,100}$ used TBSS, and 4 studies $^{8,34,44,50}$ used histogram analysis. Cross-validation of approaches can be achieved by using multiple analytic strategies. For instance, 1 article that used both a voxelwise analysis and TBSS found abnormalities in the same areas by using both techniques. ${ }^{70}$ In our review of the literature, we found that 18 articles reported using $>1$ type of analysis. ${ }^{6,14,17-19,27,34,41,46,50,55,58,63,80,86,95,98,100}$
Table 1: Most common locations of abnormal FA by ROI analysis ${ }^{\mathrm{a}}$

\begin{tabular}{lc}
\hline \multicolumn{1}{c}{ Locations } & Findings \\
\hline Corpus callosum, anterior/genu & $22^{\mathrm{b}} / 30$ \\
Corpus callosum, posterior/splenium & $21^{\mathrm{b}} / 32$ \\
Posterior limb of the internal capsule & $11 / 22$ \\
Corpus callosum, body & $10 / 18$ \\
Frontal lobe & $7 / 10$ \\
Corona radiata & $6 \mathrm{~b} / 10$ \\
Cingulum bundle & $7 / 8$ \\
Centrum semiovale & $6 / 11$ \\
Brain stem & $5 / 8$ \\
Cerebral peduncle & $5 / 7$ \\
\hline
\end{tabular}

a Values indicate the number of articles reporting abnormally low FA. Denominators represent the number of studies that assessed FA at these locations, including those that did not find abnormal FA.

${ }^{b}$ Includes articles reporting abnormally high FA. A fully referenced version of this Table is available in On-line Table 2.

Table 2: Most common locations of abnormal FA by tractography ${ }^{\mathrm{a}}$

\begin{tabular}{lc}
\hline \multicolumn{1}{c}{ Locations } & Findings \\
\hline Corpus callosum, total & $10^{\mathrm{b}} / 11$ \\
Corpus callosum, anterior/genu & $8 / 8$ \\
Corpus callosum, posterior/splenium & $7 / 8$ \\
Cingulum bundle & $6 / 10$ \\
Fornix & $5 / 7$ \\
Corpus callosum, body & $4 / 6$ \\
Fronto-occipital fasciculus & $4 / 5$ \\
Inferior longitudinal fasciculus & $4 / 5$ \\
Uncinate fasciculus & $4 / 5$ \\
Hippocampus & $3 / 3$
\end{tabular}

a Values indicate the number of articles reporting abnormally low FA. Denominators represent the number of studies that assessed FA at these locations, including those that did not find abnormal FA.

${ }^{b}$ Includes articles reporting abnormally high FA. A fully referenced version of this Table is available in On-line Table 3.

Table 3: Most common locations of abnormal FA by whole-brain analysis $^{\mathrm{a}}$

\begin{tabular}{lc}
\hline \multicolumn{1}{c}{ Locations } & Findings \\
\hline Superior longitudinal fasciculus & $7 / 25$ \\
Corpus callosum, anterior/genu & $7 / 25$ \\
Inferior longitudinal fasciculus & $7 / 25$ \\
Posterior limb of the internal capsule & $6 / 25$ \\
Fronto-occipital fasciculus & $6 / 25$ \\
Cingulum bundle & $5 / 25$ \\
Corona radiata & $5 / 25$ \\
Corpus callosum, overall & $5 / 25$ \\
Corpus callosum, body & $5 / 25$ \\
Fornix & $5 / 25$ \\
Frontal lobe & $5 / 25$ \\
Temporal lobe & $5 / 25$ \\
\hline
\end{tabular}

a Values indicate the number of articles reporting low FA in these locations. Twentyfive articles used voxelwise analysis to assess FA throughout the entire brain. Because whole-brain analyses examine all brain regions, denominators are identical for all brain regions. A fully referenced version of this Table is available in On-line Table 4.

Tables $1-3$ show the most commonly identified areas of abnormal FA in region-of-interest, tractography, and whole-brain analyses; the most commonly implicated regions are generally similar across approaches. However, they are not entirely consistent. For example, the centrum semiovale and the brain stem are locations where abnormal FA has commonly been identified by using region-of-interest analysis, but these locations are not commonly identified by using either tractography or whole-brain analysis. Additionally, the superior longitudinal fasciculus is the most commonly identified location with abnormal FA by using whole- 
Table 4: Most common locations of abnormal mean diffusivity by ROI analysis ${ }^{\mathrm{a}}$

\begin{tabular}{lc}
\hline \multicolumn{1}{c}{ Locations } & Findings \\
\hline Corpus callosum, posterior/splenium & $10^{\mathrm{b}} / 20$ \\
Corpus callosum, anterior/genu & $10 / 16$ \\
Frontal lobe & $9 / 10$ \\
White matter & $7 / 7$ \\
Thalamus & $4 / 6$ \\
\hline
\end{tabular}

a Values indicate the number of articles reporting abnormally low MD. Denominators represent the number of studies that assessed MD at these locations, including those that did not find abnormal MD.

${ }^{\mathrm{b}}$ Includes articles reporting abnormally high MD. A fully referenced version of this Table is available in On-line Table 5.

Table 5: Most common locations of abnormal mean diffusivity by tractography analysis ${ }^{\mathrm{a}}$

\begin{tabular}{lc}
\hline \multicolumn{1}{c}{ Locations } & Findings \\
\hline Corpus callosum, anterior/genu & $4 / 4$ \\
Fronto-occipital fasciculus & $4 / 5$ \\
Inferior longitudinal fasciculus & $4 / 5$ \\
Uncinate fasciculus & $4 / 4$ \\
Cingulum bundle & $3^{\mathrm{b}} / 7$
\end{tabular}

${ }^{a}$ Values indicate the number of articles reporting abnormally low MD. Denominators represent the number of studies that assessed MD at these locations, including those that did not find abnormal MD.

${ }^{\mathrm{b}}$ Includes articles reporting abnormally high MD. A fully referenced version of this Table is available in On-line Table 6.

Table 6: Most common locations of abnormal mean diffusivity by whole-brain analysis ${ }^{\mathrm{a}}$

\begin{tabular}{lc}
\multicolumn{1}{c}{ Locations } & Findings \\
\hline Cingulum bundle & $6 / 13$ \\
Corpus callosum, total & $5 / 13$ \\
Superior longitudinal fasciculus & $4 / 13$ \\
Posterior limb of the internal capsule & $4 / 13$ \\
Fronto-occipital fasciculus & $4 / 13$ \\
Frontal lobe & $4 / 13$ \\
\hline
\end{tabular}

a Values indicate the number of articles reporting abnormally increased MD in these locations. Thirteen articles used whole-brain analysis to assess MD throughout the entire brain. Because whole-brain analyses examine all brain regions, denominators are identical for all brain regions. A fully referenced version of this Table is available in On-line Table 7.

brain analysis, but this region does not appear as a common location by using either region-of-interest analysis or tractography. This discrepancy may be due to the fact that the superior longitudinal fasciculus was examined infrequently and does not necessarily indicate that it is an uncommon site of TAI. Tables 4-6 show the most commonly identified areas of abnormal MD in each of the region-of-interest, tractography, and whole-brain analyses. The corpus callosum is the most commonly identified region of abnormal FA and $\mathrm{MD}$, perhaps because it is the largest white matter tract in the brain and an important site of TBI pathology. For both reasons, the corpus callosum is commonly chosen as a target of regional analyses, a choice that may bias the net findings of the literature.

The reliability of regional analyses depends on accurate and reproducible spatial localization of ROIs or tracts across subjects. Approaches to ensuring reliability may include specific reliability and reproducibility testing when expert observers perform placement of ROIs. Alternatively, subject images may be spatially normalized to a standard template, with placement of ROIs based on the template. This latter approach ensures that region-of-interest placement is consistent among subjects; however, it depends on the robustness of the registration of the subject brains to a stan- dard space. Fourteen articles used spatial normalization to ensure

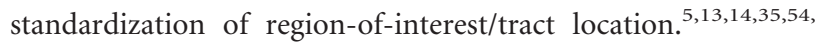
61-63,72,73,77,86,92,100 Sixty-three articles used expert manual place-

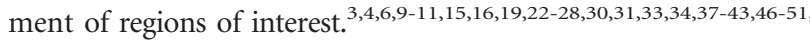
55,57,59,60,63-67,69,72,75,76,80-85,87-89,91,93-95,97-99,102 However, only 35 of 63 studies reported that they performed reliability assessments, such as inter-/intraobserver reliability. ${ }^{6,9-11,19,25,40-43}$, $46,47,49,53,55,59,60,64-67,75,76,81,83-85,87-89,91,93,94,98,99$ The studies that did report reliability testing included $63 \%$ (18/30) of articles using tractography $9,11,19,41,42,46,47,55,67,75,83-85,87-89,91,94$ but only $31 \%(19 / 61)$ of articles using region-of-interest analyses. $., 10,19,25,40,41,43,46,55,59,60,64-66,76,81,93,98,99$

In comparing articles using whole-brain approaches, $5 / 8$ of the TBSS studies found significant abnormalities, ${ }^{14,32,36,68,78}$ whereas all of the articles using voxelwise approaches identified significant differences. The greater likelihood of identifying abnormalities through voxelwise approaches as opposed to TBSS might be indicative of spurious group differences between patients and controls due to misalignment between subjects and the standard template, an issue that may be minimized by the TBSS approach. ${ }^{107}$ On the other hand, TBSS may be inherently less sensitive because the analyses are restricted to a limited white matter skeleton.

\section{SPECIFIC DIFFUSION MEASURES STUDIED}

Diffusion tensor imaging yields multiple measures at each voxel. FA describes the directional coherence of water diffusion in tissue. MD is the scalar measure of the total direction-independent diffusion within a voxel. Axial diffusivity describes diffusion along the principal axis of the diffusion ellipsoid, while radial diffusivity is an average of diffusion along its 2 minor axes. FA was the most commonly studied parameter across the studies we reviewed. Abnormally low FA is widely held to represent alterations of white matter microstructure consistent with TAI. ${ }^{108}$ Elevations of FA have been much less frequently reported (see below). Although some authors have hypothesized that abnormally high FA represents cytotoxic edema, ${ }^{54}$ the mechanistic basis of abnormally high FA remains uncertain.

FA was examined in all studies. All 100 articles, except for $4^{6,54,89,96}$ (which showed elevated FA), described findings of low FA in subjects with TBI, regardless of the time of injury and across the spectrum of injury severity. MD is the next most commonly reported parameter $(n=51)$. $3,9,10,13-17,19,21,22,25,28,29,33-40,42$, 43,48-50,55,56,59-63,66,67,69,71,72,76,77,81,83,90,91,93,95,96,100-102 Articles that examined parameters other than FA almost always identified areas of abnormal FA and then assessed other parameters within these regions. One article initially identified loci of abnormal MD and then assessed FA in the regions of abnormal MD. ${ }^{20}$ Axial diffusivity and radial diffusivity were much less commonly assessed $(n=$ 18). ${ }^{3,8,11,13,14,20,22,36-38,49,54,56,63,66,76,81,89}$ Only 2 articles reported radial diffusivity and not axial diffusivity. ${ }^{29,66}$ One article used a predictive model in which all 4 measures were incorporated. $^{65}$

While FA is a useful summary measure, more detailed information regarding diffusional uniformity, potentially obtainable through study of eigenvalue measures, may be important for diagnosis and outcome prediction and especially for understanding 
Table 7: Relationship of DTI metrics to cognitive outcome measures ${ }^{\mathrm{a}}$

\begin{tabular}{|c|c|c|c|c|c|c|c|c|}
\hline $\begin{array}{c}\text { DTI } \\
\text { Measure } \\
\end{array}$ & Correlation & Attention & $\begin{array}{l}\text { Executive } \\
\text { Function }\end{array}$ & Memory & Motor & $\begin{array}{c}\text { Psychomotor/ } \\
\text { Processing Speed }\end{array}$ & Visuospatial & IQ \\
\hline \multirow[t]{3}{*}{ FA } & Positive correlation & 11 & 9 & 14 & 4 & 5 & 4 & 2 \\
\hline & Negative correlation & 6 & 5 & 2 & 0 & 2 & 0 & 0 \\
\hline & No correlation & 2 & 6 & 6 & 1 & 1 & 0 & 6 \\
\hline \multirow[t]{3}{*}{ MD } & Positive correlation & 3 & 2 & 2 & 0 & 0 & 1 & 0 \\
\hline & Negative correlation & 4 & 6 & 7 & 0 & 1 & 3 & 0 \\
\hline & No correlation & 1 & 4 & 3 & 1 & 2 & 0 & 0 \\
\hline
\end{tabular}

Note:- IQ indicates intelligence quotient.

${ }^{a}$ Total number of articles assessing relationships between DTI measures and cognitive outcomes. Cognitive-outcome measures have been categorized as 7 domains (top row). Articles are classified as reporting positive correlation, negative correlation, or no correlation. Positive correlation indicates a correlation coefficient greater than zero. Negative correlation indicates a correlation coefficient less than zero. No correlation includes articles that reported analyzing relationships between the DTI measures and cognitive outcomes within a domain but either reported finding no correlation (correlation coefficient equal to zero) or a correlation with a $P$ value $>.05$. A fully referenced version of this Table is available in On-line Table 8.

pathologic mechanisms of TBI in humans. Eigenvalue measures implicate specific pathologic mechanisms in animal models of TBI and other types of brain injury. ${ }^{2}$ Including eigenvalue measures in a future study would facilitate a transitional bridge from animal to human studies and enable more informed targeting of novel interventions in a patient group with few therapeutic options.

\section{BRAIN REGIONS}

Brain regions examined varied greatly among the articles. We systematically tabulated the location of abnormal DTI measures (FA and MD separately) across all articles (Tables 1-6). To effectively summarize a large number of regions, we list only the top 10 most commonly identified regions of abnormal FA and the top 5 most commonly identified regions of abnormal MD via regionof-interest, tractography, and whole-brain analyses. When tabulating abnormal regions, it is important to recognize the relationship between study design and detection-that is, regional analyses can only detect a region as abnormal if the study design specifically examines that region. Whole-brain analyses, on the other hand, are positioned to detect all abnormal areas in the brain. Thus, the number of studies reporting an area as abnormal is influenced by the study design. Nonetheless, overall we found a large degree of consistency between studies regardless of the method of analysis. The corpus callosum, frontal lobe, internal capsule, and cingulum are among the most commonly identified regions of abnormality in DTI studies of TBI, perhaps because these structures are particularly vulnerable to injury due to their anatomic relationship to the skull and other structures such as the falx cerebri. These findings are almost entirely based on group comparisons and, therefore, do not necessarily reflect the distribution of injuries in the individual.

\section{FUNCTIONAL OUTCOMES AFTER TBI}

Seventy-two of 100 articles examined outcomes associated with

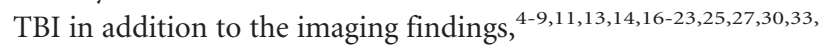
36-43,45-47,49-59,61,62,64-68,70,71,74-76,78,80-82,84,87-94,96,98,101,102 and

almost all of these 72 articles reported specifically on the association of DTI findings with the outcomes. Most surprising however, 3 articles, though they reported outcomes, did not report analysis of the relationship between the outcome measures and DTI. ${ }^{27,35,56}$ Fifty-one of the 72 articles assessed neuropsychological outcomes. ${ }^{5-7,9-14,16-19,22,23,25,27,30,36-39,41-43 \text {, }}$ 45,46,50-52,54,57-60,64,65,67,68,74,75,80,81,88,90-94,96,102 Eighteen of 72 used a global outcome measure (eg, Glasgow Outcomes
Table 8: Relationship of DTI metrics to general clinical assessments $^{\mathrm{a}}$

\begin{tabular}{|c|c|c|c|c|}
\hline $\begin{array}{c}\text { DTI } \\
\text { Measure }\end{array}$ & Correlation & $\begin{array}{c}\text { Global } \\
\text { Outcome } \\
\text { Measures }\end{array}$ & GCS & $\begin{array}{l}\text { Postconcussion } \\
\text { Symptoms }\end{array}$ \\
\hline \multirow[t]{3}{*}{ FA } & Positive correlation & 11 & 5 & 3 \\
\hline & Negative correlation & 4 & 1 & 3 \\
\hline & No correlation & 3 & 8 & 6 \\
\hline \multirow[t]{3}{*}{ MD } & Positive correlation & 1 & 1 & 1 \\
\hline & Negative correlation & 5 & 4 & 2 \\
\hline & No correlation & 0 & 0 & 1 \\
\hline
\end{tabular}

${ }^{a}$ Total number of articles assessing relationships between DTI measures and global outcome measures (see "Functional Outcomes after TBI"), GCS, or postconcussive symptoms. Articles are classified as reporting positive correlation, negative correlation, or no correlation. Positive correlation indicates a correlation coefficient greater than zero. Negative correlation indicates a correlation coefficient less than zero. No correlation includes articles that reported analyzing relationships between the DTI measure and cognitive outcomes within a domain but either reported finding no correlation (correlation coefficient equal to zero) or a correlation with a $P$ value $>.05$. A fully referenced version of this Table is available in On-line Table 9.

Scale, Coma Recovery Scale, Mini-Mental Status Examination), ${ }^{4,16,22,33,42,50,57-59,63,66,70,76,80-82,84,88} 14$ articles reported correlation with the GCS, $4,5,7,8,22,33,38,42,61,62,66,87,91,988$ articles correlated FA with intelligence quotient, ,5,14,16,22,54,58,80,92 and 12 articles examined the relationship between DTI and postconcussive symptoms (eg, headaches, visual disturbances, cognitive symptoms) and/or mood disorders. ${ }^{6,20,23,40,53,54,78,80,81,89,92,99}$ Several articles reported the association of DTI with other patient assessments as surrogate outcome measures. These measures included electroencephalography, ${ }^{79} \mathrm{fMRI},{ }^{10,63,69,75,90,100} \mathrm{MR}$ spectroscopy, ${ }^{5,82}$ regional brain volumes, ${ }^{85}$ and motor-evoked potentials. ${ }^{97}$

Of the articles that correlated DTI metrics with outcome, we found considerable heterogeneity among studies with respect to the specific outcome measures used. Choice of specific neuropsychological tests was most variable, with only a few articles reporting the same measure (range, 1-19; mean, 1.8; median, 1.5). To summarize the data from these studies, we divided the various outcome measures into domains, including the following: attention, executive function, memory, motor function, psychomotor/ processing speed, visuospatial function, global outcome, GCS, intelligence quotient, and postconcussive symptoms. Tables 7 and 8 summarize the significant associations reported between DTI metrics and these outcome categories.

In addition to heterogeneity of the outcome measures used, additional variability (eg, brain region examined, analysis type, and DTI metric assessed) among studies examining the same out- 
come domain further complicated summary of the literature. The average number of studies examining the same outcome domain was 15 (range, 6-29; Tables 7 and 8). However, even among articles examining the same domain, results are inconsistent.

Discordance in the results of outcome studies can, perhaps, be attributed to several issues. Although studies may examine the same domain, they typically vary in the choice of the specific measure/instrument used. Because the sensitivity and specificity of any 2 measures, though designed to test the same cognitive domain, for instance, will differ, ${ }^{109}$ results of studies relating imaging to these differing outcome measures will potentially differ between the 2 studies as well. Moreover, the severity of TBI might determine the extent to which a patient experiences impairment in cognition or other adverse outcomes. As a result, comparison between studies including different injury severities might not be appropriate. Finally, impairment secondary to TBI, particularly mild TBI, can be subtle and, therefore, escape detection by using some formal testing tools. Perhaps the most salient message to be derived from this segment of the literature is that standardization of outcome measures and study design is essential to future meaningful study of TBI. ${ }^{110}$

\section{ASSESSMENT OF INDIVIDUAL PATIENTS WITH TBI}

The heterogeneity of injury mechanisms that cause TBI is likely best captured in studies assessing individual patients with TBI; group comparisons are inherently insensitive to interindividual variation, which is a hallmark of TBI. All of the articles included in this review report group analyses of patients with TBI. However, $>35$ additional articles report the use of DTI in individual TBI cases (eg, Gold and Lipton ${ }^{111}$ ). Several of the articles included in this review reported assessment of individual patients in addition to their group analyses. Two articles examined whole-brain white matter histograms of individual patients, ${ }^{8,50}$ but only 1 reported results at the single-subject level, finding the distribution of FA in patients with TBI to be skewed toward lower FA in comparison with controls. ${ }^{8}$ Three articles applied a tractography approach to individual subjects, ${ }^{31,58,63}$ while 2 articles applied the whole-brain approach to individuals. ${ }^{44,50}$ Assessment of individual patients with TBI is important to the characterization of outliers, who might differ from the group in terms of extent and spatial distribution of injury, and is a prerequisite to clinical use of DTI in evaluating patients with TBI.

\section{IMPLICATIONS, LIMITATIONS, AND POSSIBILITIES}

DTI has been studied extensively as a tool for identification of brain abnormalities related to TBI and to understand the relationship of these brain abnormalities to other clinical features of the disorder. During the past decade, the number of such studies has risen exponentially and continues to increase with no sign of abatement. A unifying theme can be deduced from this large body of research: DTI is an extremely useful and robust tool for the detection of TBI-related brain abnormalities. The overwhelming consensus of these studies is that low white matter FA is characteristic of TBI. This finding is consistent across almost all the articles we reviewed, despite significant variability in patient demographics, modest differences in data acquisition parameters, and a multiplicity of data analysis techniques. This consistency across studies attests to the robustness of DTI as a measure of brain injury in TBI. The finding of significant differences in FA histograms that pool all white matter voxels across the whole brain is particularly compelling, indicating that a substantial portion of the hundreds of thousands of voxels in the image datasets are abnormal.

We also found an overwhelming consensus that imaging abnormalities detected with DTI are associated with important clinical outcomes. This further validates DTI as a meaningful measure of clinically important brain injury. However, heterogeneity among the outcome measures that have been reported limits our ability to draw direct generalizable connections between DTI abnormalities at specific brain locations and specific outcomes. The greatest degree of variability among the studies we reviewed was in the choice of outcome measures. As others have suggested, an important priority for future studies of TBI should be the use of standardized approaches, particularly standardization of outcome measures. ${ }^{112}$ Additionally, more high-quality longitudinal studies are needed to extend the power of DTI, from identifying patients with TBI at cross-section to accurately predicting future clinical status.

By far, FA was the DTI measure used most commonly across the studies we reviewed. Too few articles reported analyses of eigenvalues to permit meaningful inferences regarding the role of eigenvalue findings in the assessment of TBI at this time. This is an important area of deficiency because preclinical studies indicate that differential effects on eigenvalue measures can separate pathologic mechanisms. Thus, more detailed study of the full palette of metrics available from DTI is a great area for future study.

The variety of data analysis approaches applied across the studies we reviewed presents a significant obstacle to summary of the data and limits the inferences that can be made on the basis of the literature as a whole. It is primarily on the basis of this factor that we determined that meta-analysis methods would not be appropriate for assessment of this literature. Studies reporting regional analyses might be considered comparable on the basis of similarity of brain regions tested across multiple studies. However, the precise spatial location represented by a given region-ofinterest, tract, or brain structure descriptors may vary significantly among studies on the basis of the methods, criteria, and raters used to define ROIs. Intra- and inter-rater variability can confound inferences based on these studies. While reliability testing can and should be performed to verify the reproducibility of region-of-interest placement, only a minority of studies did so.

Whole-brain analyses perhaps offer the greatest promise for pooling of results across studies, provided that the studies normalize their image data to the same brain template. Variability in the brain atlases used for spatial normalization and inconsistent reporting of coordinates for abnormalities in the studies we reviewed limit such cross-study comparison at this time.

All articles captured by our review used group analyses, though several also incorporated assessments of individual patients with TBI. Group-analysis approaches are powerful means for improving statistical power. However, the use of a group analysis limits the study to detection of abnormalities that occur in the same location in all patients. The fact that robust group effects have been reproduced in studies of DTI in patients with TBI is consistent with long-standing concepts that identify certain brain regions as particularly susceptible to TAI. Intersubject differences in the mechanism of injury as well as other biomechanical factors

AJNR Am J Neuroradiol 34:2064-74 Nov 2013 www.ajnr.org 
such as head and body composition make it highly probable that despite some commonalities, many areas of injury will differ among patients. Further application of individualized assessments of regional brain injury is thus needed to realize the full potential of DTI as a research and clinical tool.

On the basis of our analysis of the current literature, we suggest that important focus areas for future study should include larger longitudinal studies, incorporation of multiple outcome measures in statistical models that account for the complexity inherent in TBI populations, and assessment of interindividual differences. Standardization across centers, specifically with regard to data acquisition parameters, data analysis techniques, and the specific outcome measures assessed, promises to greatly increase the yield of such studies.

In summary, DTI provides a robust measure of clinically important TAI at cross-section, despite the variability inherent in characteristics of patients with TBI and injury mechanisms as well as study differences in data acquisition and analysis methods. Larger longitudinal studies will be essential for the evaluation of DTI as a prognostic biomarker in TBI. More detailed assessment of DTI metrics and translational imaging studies should be undertaken to link pathophysiologic mechanisms in animal models to important clinical outcomes in patients. Together, these approaches promise to realize the full potential of DTI to improve diagnosis and treatment of patients with TBI.

\section{ACKNOWLEDGMENTS}

We thank Tova M. Gardin for graphic arts assistance.

Disclosures: Molly E. Zimmerman—UNRELATED: Grants/Grants Pending: National Institutes of Health (NIH)/National Institute on Aging, ${ }^{*}$ Alzheimer's Association,* Bristol-Myers Squibb.* Michael L. Lipton—UNRELATED: Grants/Grants Pending: $\mathrm{NIH},{ }^{\star}$ Dana Foundation, ${ }^{*}$ Resurrecting Lives Foundation, ${ }^{*}$ Patents (planned, pending, or issued): Einstein patent pending, ${ }^{*}$ Comments: for data analysis techniques. *Money paid to the institution.

\section{REFERENCES}

1. Niogi SN, Mukherjee P. Diffusion tensor imaging of mild traumatic brain injury. J Head Trauma Rehabil 2010;25:241-55

2. Mac Donald CL, Dikranian K, Bayly P, et al. Diffusion tensor imaging reliably detects experimental traumatic axonal injury and indicates approximate time of injury. J Neurosci 2007;27:11869-76

3. Arfanakis K, Haughton VM, Carew JD, et al. Diffusion tensor MR imaging in diffuse axonal injury. AJNR Am J Neuroradiol 2002;23: 794-802

4. Akpinar E, Koroglu M, Ptak T. Diffusion tensor MR imaging in pediatric head trauma. J Comput Assist Tomogr 2007;31:657-61

5. Babikian T, Marion SD, Copeland S, et al. Metabolic levels in the corpus callosum and their structural and behavioral correlates after moderate to severe pediatric TBI. J Neurotrauma 2010;27: 473-81

6. Bazarian JJ, Zhong J, Blyth B, et al. Diffusion tensor imaging detects clinically important axonal damage after mild traumatic brain injury: a pilot study. J Neurotrauma 2007;24:1447-59

7. Bendlin BB, Ries ML, Lazar M, et al. Longitudinal changes in patients with traumatic brain injury assessed with diffusion-tensor and volumetric imaging. Neuroimage 2008;42:503-14

8. Benson RR, Meda SA, Vasudevan S, et al. Global white matter analysis of diffusion tensor images is predictive of injury severity in traumatic brain injury. J Neurotrauma 2007;24:446-59

9. Bigler ED, McCauley SR, Wu TC, et al. The temporal stem in traumatic brain injury: preliminary findings. Brain Imaging Behav 2010;4:270-82
10. Bonnelle V, Leech R, Kinnunen KM, et al. Default mode network connectivity predicts sustained attention deficits after traumatic brain injury. J Neurosci 2011;31:13442-51

11. Caeyenberghs K, Leemans A, Coxon J, et al. Bimanual coordination and corpus callosum microstructure in young adults with traumatic brain injury: a diffusion tensor imaging study. $\mathrm{J} \mathrm{Neu}$ rotrauma 2011;28:897-913

12. Caeyenberghs K, Leemans A, Geurts M, et al. Correlations between white matter integrity and motor function in traumatic brain injury patients. Neurorehabil Neural Repair 2011;25:492-502

13. Caeyenberghs K, Leemans A, Geurts M, et al. Brain-behavior relationships in young traumatic brain injury patients: DTI metrics are highly correlated with postural control. Hum Brain Mapp 2010; 31:992-1002

14. Caeyenberghs K, Leemans A, Geurts M, et al. Brain-behavior relationships in young traumatic brain injury patients: fractional anisotropy measures are highly correlated with dynamic visuomotor tracking performance. Neuropsychologia 2010;48:1472-82

15. Chang MC, Jang SH. Corpus callosum injury in patients with diffuse axonal injury: a diffusion tensor imaging study. NeuroRehabilitation 2010;26:339-45

16. Chang MC, Kim SH, Kim OL, et al. The relation between fornix injury and memory impairment in patients with diffuse axonal injury: a diffusion tensor imaging study. NeuroRehabilitation 2010; 26:347-53

17. Cho HK, Hong JH, Kim SH, et al. Clinical usefulness of diffusion tensor imaging in patients with transtentorial herniation following traumatic brain injury. Brain Inj 2011;25:1005-09

18. Choi GS, Kim OL, Kim SH, et al. Classification of cause of motor weakness in traumatic brain injury using diffusion tensor imaging. Arch Neurol 2012;69:363-67

19. Christidi F, Bigler ED, McCauley SR, et al. Diffusion tensor imaging of the perforant pathway zone and its relation to memory function in patients with severe traumatic brain injury. J Neurotrauma 2011;28:711-25

20. Chu Z, Wilde EA, Hunter JV, et al. Voxel-based analysis of diffusion tensor imaging in mild traumatic brain injury in adolescents. AJNR Am J Neuroradiol 2010;31:340-46

21. Cubon VA, Putukian M, Boyer C, et al. A diffusion tensor imaging study on the white matter skeleton in individuals with sports-related concussion. J Neurotrauma 2011;28:189-201

22. Ewing-Cobbs L, Prasad MR, Swank P, et al. Arrested development and disrupted callosal microstructure following pediatric traumatic brain injury: relation to neurobehavioral outcomes. Neuroimage 2008;42:1305-15

23. Geary EK, Kraus MF, Pliskin NH, et al. Verbal learning differences in chronic mild traumatic brain injury. J Int Neuropsychol Soc 2010; 16:506-16

24. Greenberg G, Mikulis DJ, Ng K, et al. Use of diffusion tensor imaging to examine subacute white matter injury progression in moderate to severe traumatic brain injury. Arch Phys Med Rehabil 2008; 89:S45-50

25. Grossman EJ, Ge Y, Jensen JH, et al. Thalamus and cognitive impairment in mild traumatic brain injury: a diffusional kurtosis imaging study. J Neurotrauma 2012;29:2318-27

26. Gupta RK, Saksena S, Agarwal A, et al. Diffusion tensor imaging in late posttraumatic epilepsy. Epilepsia 2005;46:1465-71

27. Hanten G, Wilde EA, Menefee DS, et al. Correlates of social problem solving during the first year after traumatic brain injury in children. Neuropsychology 2008;22:357-70

28. Hartikainen KM, Waljas M, Isoviita T, et al. Persistent symptoms in mild to moderate traumatic brain injury associated with executive dysfunction. J Clin Exp Neuropsychol 2010;32:767-74

29. Henry LC, Tremblay J, Tremblay S, et al. Acute and chronic changes in diffusivity measures after sports concussion. J Neurotrauma 2011;28:2049-59

30. Holli KK, Waljas M, Harrison L, et al. Mild traumatic brain injury 
tissue texture analysis correlated to neuropsychological and DTI findings. Acad Radiol 2010;17:1096-102

31. Hong JH, Kim OL, Kim SH, et al. Cerebellar peduncle injury in patients with ataxia following diffuse axonal injury. Brain Res Bull 2009;80:30-35

32. Huang MX, Theilmann RJ, Robb A, et al. Integrated imaging approach with MEG and DTI to detect mild traumatic brain injury in military and civilian patients. J Neurotrauma 2009;26:1213-26

33. Huisman TA, Schwamm LH, Schaefer PW, et al. Diffusion tensor imaging as potential biomarker of white matter injury in diffuse axonal injury. AJNR Am J Neuroradiol 2004;25:370-76

34. Inglese M, Makani S, Johnson G, et al. Diffuse axonal injury in mild traumatic brain injury: a diffusion tensor imaging study. J Neurosurg 2005;103:298-303

35. Kennedy MR, Wozniak JR, Muetzel RL, et al. White matter and neurocognitive changes in adults with chronic traumatic brain injury. J Int Neuropsychol Soc 2009;15:130-36

36. Kinnunen KM, Greenwood R, Powell JH, et al. White matter damage and cognitive impairment after traumatic brain injury. Brain 2011;134:449-63

37. Kraus MF, Susmaras T, Caughlin BP, et al. White matter integrity and cognition in chronic traumatic brain injury: a diffusion tensor imaging study. Brain 2007;130:2508-19

38. Kumar R, Gupta RK, Husain M, et al. Comparative evaluation of corpus callosum DTI metrics in acute mild and moderate traumatic brain injury: its correlation with neuropsychometric tests. Brain Inj 2009;23:675-85

39. Kumar R, Husain M, Gupta RK, et al. Serial changes in the white matter diffusion tensor imaging metrics in moderate traumatic brain injury and correlation with neuro-cognitive function. $\mathrm{JNeu}$ rotrauma 2009;26:481-95

40. Lange RT, Iverson GL, Brubacher JR, et al. Diffusion tensor imaging findings are not strongly associated with postconcussional disorder 2 months following mild traumatic brain injury. J Head Trauma Rehabil 2012;27:188-98

41. Levin HS, Wilde E, Troyanskaya M, et al. Diffusion tensor imaging of mild to moderate blast-related traumatic brain injury and its sequelae. J Neurotrauma 2010;27:683-94

42. Levin HS, Wilde EA, Chu Z, et al. Diffusion tensor imaging in relation to cognitive and functional outcome of traumatic brain injury in children. J Head Trauma Rehabil 2008;23:197-208

43. Levin HS, Wilde EA, Hanten G, et al. Mental state attributions and diffusion tensor imaging after traumatic brain injury in children. Dev Neuropsychol 2011;36:273-87

44. Lipton ML, Gellella E, Lo C, et al. Multifocal white matter ultrastructural abnormalities in mild traumatic brain injury with cognitive disability: a voxel-wise analysis of diffusion tensor imaging. J Neurotrauma 2008;25:1335-42

45. Lipton ML, Gulko E, Zimmerman ME, et al. Diffusion tensor imaging implicates prefrontal axonal injury in executive function impairment following mild traumatic brain injury. Radiology 2009;252:816-24

46. Little DM, Kraus MF, Joseph J, et al. Thalamic integrity underlies executive dysfunction in traumatic brain injury. Neurology 2010; 74:558-64

47. Ljungqvist J, Nilsson D, Ljungberg M, et al. Longitudinal study of the diffusion tensor imaging properties of the corpus callosum in acute and chronic diffuse axonal injury. Brain Inj 2011;25:370-78

48. Lo C, Shifteh K, Gold T, et al. Diffusion tensor imaging abnormalities in patients with mild traumatic brain injury and neurocognitive impairment. J Comput Assist Tomogr 2009;33:293-97

49. Mac Donald CL, Johnson AM, Cooper D, et al. Detection of blastrelated traumatic brain injury in U.S. military personnel. $N$ Engl J Med 2011;364:2091-100

50. Marquez de la Plata CD, Yang FG, Wang JY, et al. Diffusion tensor imaging biomarkers for traumatic axonal injury: analysis of three analytic methods. J Int Neuropsychol Soc 2011;17:24-35

51. Maruta J, Suh M, Niogi SN, et al. Visual tracking synchronization as a metric for concussion screening. J Head Trauma Rehabil 2010; 25:293-305

52. Matsushita M, Hosoda K, Naitoh Y, et al. Utility of diffusion tensor imaging in the acute stage of mild to moderate traumatic brain injury for detecting white matter lesions and predicting long-term cognitive function in adults. J Neurosurg 2011;115:130-39

53. Matthews SC, Strigo IA, Simmons AN, et al. A multimodal imaging study in U.S. veterans of Operations Iraqi and Enduring Freedom with and without major depression after blast-related concussion. Neuroimage 2011;54(suppl 1):S69-75

54. Mayer AR, Ling J, Mannell MV, et al. A prospective diffusion tensor imaging study in mild traumatic brain injury. Neurology 2010;74: $643-50$

55. McCauley SR, Wilde EA, Bigler ED, et al. Diffusion tensor imaging of incentive effects in prospective memory after pediatric traumatic brain injury. J Neurotrauma 2011;28:503-16

56. Messé A, Caplain S, Paradot G, et al. Diffusion tensor imaging and white matter lesions at the subacute stage in mild traumatic brain injury with persistent neurobehavioral impairment. Hum Brain Mapp 2011;32:999-1011

57. Miles L, Grossman RI, Johnson G, et al. Short-term DTI predictors of cognitive dysfunction in mild traumatic brain injury. Brain Inj 2008;22:115-22

58. Nakayama N, Okumura A, Shinoda J, et al. Evidence for white matter disruption in traumatic brain injury without macroscopic lesions. J Neurol Neurosurg Psychiatry 2006;77:850-55

59. Newcombe V, Chatfield D, Outtrim J, et al. Mapping traumatic axonal injury using diffusion tensor imaging: correlations with functional outcome. PloS One 2011;6:e19214

60. Newcombe VF, Outtrim JG, Chatfield DA, et al. Parcellating the neuroanatomical basis of impaired decision-making in traumatic brain injury. Brain 2011;134:759-68

61. Newcombe VF, Williams GB, Nortje J, et al. Concordant biology underlies discordant imaging findings: diffusivity behaves differently in grey and white matter post acute neurotrauma. Acta Neurochir Suppl 2008;102:247-51

62. Newcombe VF, Williams GB, Nortje J, et al. Analysis of acute traumatic axonal injury using diffusion tensor imaging. Br J Neurosurg 2007;21:340-48

63. Newcombe VF, Williams GB, Scoffings D, et al. Aetiological differences in neuroanatomy of the vegetative state: insights from diffusion tensor imaging and functional implications. J Neurol Neurosurg Psychiatry 2010;81:552-61

64. Niogi SN, Mukherjee P, Ghajar J, et al. Extent of microstructural white matter injury in postconcussive syndrome correlates with impaired cognitive reaction time: a $3 \mathrm{~T}$ diffusion tensor imaging study of mild traumatic brain injury. AJNR Am J Neuroradiol 2008; 29:967-73

65. Niogi SN, Mukherjee P, Ghajar J, et al. Structural dissociation of attentional control and memory in adults with and without mild traumatic brain injury. Brain 2008;131:3209-21

66. Oni MB, Wilde EA, Bigler ED, et al. Diffusion tensor imaging analysis of frontal lobes in pediatric traumatic brain injury. $J$ Child Neurol 2010;25:976-84

67. Pal D, Gupta RK, Agarwal S, et al. Diffusion tensor tractography indices in patients with frontal lobe injury and its correlation with neuropsychological tests. Clin Neurol Neurosurg 2012;114:564-71

68. Palacios EM, Fernandez-Espejo D, Junque C, et al. Diffusion tensor imaging differences relate to memory deficits in diffuse traumatic brain injury. BMC Neurol 2011;11:24

69. Palmer HS, Garzon B, Xu J, et al. Reduced fractional anisotropy does not change the shape of the hemodynamic response in survivors of severe traumatic brain injury. J Neurotrauma 2010;27: $853-62$

70. Perlbarg V, Puybasset L, Tollard E, et al. Relation between brain lesion location and clinical outcome in patients with severe traumatic brain injury: a diffusion tensor imaging study using voxelbased approaches. Hum Brain Mapp 2009;30:3924-33 
71. Porto L, Jurcoane A, Margerkurth J, et al. Morphometry and diffusion MR imaging years after childhood traumatic brain injury. Eur J Paediatr Neurol 2011;15:493-501

72. Rutgers DR, Fillard P, Paradot G, et al. Diffusion tensor imaging characteristics of the corpus callosum in mild, moderate, and severe traumatic brain injury. AJNR Am J Neuroradiol 2008;29:1730-35

73. Rutgers DR, Toulgoat F, Cazejust J, et al. White matter abnormalities in mild traumatic brain injury: a diffusion tensor imaging study. AJNR Am J Neuroradiol 2008;29:514-19

74. Salmond CH, Menon DK, Chatfield DA, et al. Diffusion tensor imaging in chronic head injury survivors: correlations with learning and memory indices. Neuroimage 2006;29:117-24

75. Scheibel RS, Newsome MR, Wilde EA, et al. Brain activation during a social attribution task in adolescents with moderate to severe traumatic brain injury. Soc Neurosci 2011;6:582-98

76. Sidaros A, Engberg AW, Sidaros K, et al. Diffusion tensor imaging during recovery from severe traumatic brain injury and relation to clinical outcome: a longitudinal study. Brain 2008;131:559-72

77. Singh $M$, Jeong J, Hwang D, et al. Novel diffusion tensor imaging methodology to detect and quantify injured regions and affected brain pathways in traumatic brain injury. Magn Reson Imaging 2010;28:22-40

78. Smits M, Houston GC, Dippel DW, et al. Microstructural brain injury in post-concussion syndrome after minor head injury. $\mathrm{Neu}$ roradiology 2011;53:553-63

79. Sponheim SR, McGuire KA, Kang SS, et al. Evidence of disrupted functional connectivity in the brain after combat-related blast injury. Neuroimage 2011;54(suppl 1):S21-29

80. Sugiyama K, Kondo T, Oouchida Y, et al. Clinical utility of diffusion tensor imaging for evaluating patients with diffuse axonal injury and cognitive disorders in the chronic stage. J Neurotrauma 2009;26:1879-90

81. Tasker RC, Gunn Westland A, White DK, et al. Corpus callosum and inferior forebrain white matter microstructure are related to functional outcome from raised intracranial pressure in child traumatic brain injury. Dev Neurosci 2010;32:374-84

82. Tollard E, Galanaud D, Perlbarg V, et al. Experience of diffusion tensor imaging and $1 \mathrm{H}$ spectroscopy for outcome prediction in severe traumatic brain injury: preliminary results. Crit Care Med 2009;37:1448-55

83. Wang JY, Bakhadirov K, Abdi H, et al. Longitudinal changes of structural connectivity in traumatic axonal injury. Neurology 2011;77:818-26

84. Wang JY, Bakhadirov K, Devous MD, Sr., et al. Diffusion tensor tractography of traumatic diffuse axonal injury. Arch Neurol 2008;65: 619-26

85. Warner MA, Marquez de la Plata C, Spence J, et al. Assessing spatial relationships between axonal integrity, regional brain volumes, and neuropsychological outcomes after traumatic axonal injury. J Neurotrauma 2010;27:2121-30

86. Wei CW, Tharmakulasingam J, Crawley A, et al. Use of diffusion-tensor imaging in traumatic spinal cord injury to identify concomitant traumatic brain injury. Arch Phys Med Rehabil 2008;89:S85-91

87. Wilde EA, Bigler ED, Haider JM, et al. Vulnerability of the anterior commissure in moderate to severe pediatric traumatic brain injury. J Child Neurol 2006;21:769-76

88. Wilde EA, Chu Z, Bigler ED, et al. Diffusion tensor imaging in the corpus callosum in children after moderate to severe traumatic brain injury. J Neurotrauma 2006;23:1412-26

89. Wilde EA, McCauley SR, Hunter JV, et al. Diffusion tensor imaging of acute mild traumatic brain injury in adolescents. Neurology 2008;70:948-55

90. Wilde EA, Newsome MR, Bigler ED, et al. Brain imaging correlates of verbal working memory in children following traumatic brain injury. Int J Psychophysiol 2011;82:86-96

91. Wilde EA, Ramos MA, Yallampalli R, et al. Diffusion tensor imaging of the cingulum bundle in children after traumatic brain injury. Dev Neuropsychol 2010;35:333-51
92. Wozniak JR, Krach L, Ward E, et al. Neurocognitive and neuroimaging correlates of pediatric traumatic brain injury: a diffusion tensor imaging (DTI) study. Arch Clin Neuropsychol 2007;22:555-68

93. Wu TC, Wilde EA, Bigler ED, et al. Longitudinal changes in the corpus callosum following pediatric traumatic brain injury. Dev Neurosci 2010;32:361-73

94. Wu TC, Wilde EA, Bigler ED, et al. Evaluating the relationship between memory functioning and cingulum bundles in acute mild traumatic brain injury using diffusion tensor imaging. $\mathrm{J} \mathrm{Neu-}$ rotrauma 2010;27:303-07

95. Xu J, Rasmussen IA, Lagopoulos J, et al. Diffuse axonal injury in severe traumatic brain injury visualized using high-resolution diffusion tensor imaging. J Neurotrauma 2007;24:753-65

96. Yallampalli R, Wilde EA, Bigler ED, et al. Acute white matter differences in the fornix following mild traumatic brain injury using diffusion tensor imaging. J Neuroimaging 2013;23:224-27

97. Yasokawa YT, Shinoda J, Okumura A, et al. Correlation between diffusion-tensor magnetic resonance imaging and motor-evoked potential in chronic severe diffuse axonal injury. J Neurotrauma 2007;24:163-73

98. Yuan W, Holland SK, Schmithorst VJ, et al. Diffusion tensor MR imaging reveals persistent white matter alteration after traumatic brain injury experienced during early childhood. AJNR Am J Neuroradiol 2007;28:1919-25

99. Yurgelun-Todd DA, Bueler CE, McGlade EC, et al. Neuroimaging correlates of traumatic brain injury and suicidal behavior. $J$ Head Trauma Rehabil 2011;26:276-89

100. Zhang K, Johnson B, Pennell D, et al. Are functional deficits in concussed individuals consistent with white matter structural alterations: combined FMRI \& DTI study. Exp Brain Res 2010;204:57-70

101. Hong JH, Jang SH, Kim OL, et al. Neuronal loss in the medial cholinergic pathway from the nucleus basalis of Meynert in patients with traumatic axonal injury: a preliminary diffusion tensor imaging study. J Head Trauma Rehabil 2012;27:172-76

102. Kumar R, Saksena S, Husain M, et al. Serial changes in diffusion tensor imaging metrics of corpus callosum in moderate traumatic brain injury patients and their correlation with neuropsychometric tests: a 2-year follow-up study. J Head Trauma Rehabil 2010;25:31-42

103. Rosenbaum SB, Lipton ML. Embracing chaos: the scope and importance of clinical and pathological heterogeneity in $\mathrm{mTBI}$. Brain Imaging Behav 2012;6:255-82

104. Kashluba S, Hanks RA, Casey JE, et al. Neuropsychologic and functional outcome after complicated mild traumatic brain injury. Arch Phys Med Rehabil 2008;89:904-11

105. Burdette JH, Durden DD, Elster AD, et al. High b-value diffusion-weighted MRI of normal brain. J Comput Assist Tomogr 2001;25:515-19

106. Dudink J, Larkman DJ, Kapellou O, et al. High b-value diffusion tensor imaging of the neonatal brain at 3T. AJNR Am J Neuroradiol 2008:29:1966-72

107. Smith SM, Jenkinson M, Johansen-Berg H, et al. Tract-based spatial statistics: voxelwise analysis of multi-subject diffusion data. $\mathrm{Neu}$ roimage 2006;31:1487-505

108. Shenton ME, Hamoda HM, Schneiderman JS, et al. A review of magnetic resonance imaging and diffusion tensor imaging findings in mild traumatic brain injury. Brain Imaging Behav 2012;6:137-92

109. Cicerone KD, Azulay J. Diagnostic utility of attention measures in postconcussion syndrome. Clin Neuropsychol 2002;16:280-89

110. Duhaime AC, Holshouser B, Hunter JV, et al. Common data elements for neuroimaging of traumatic brain injury: pediatric considerations. J Neurotrauma 2012;29:629-33

111. Gold MM, Lipton ML. Neurological picture: diffusion tractography of axonal degeneration following shear injury. J Neurol Neurosurg Psychiatry 2008;79:1374-75

112. Duhaime AC, Gean AD, Haacke EM, et al. Common data elements in radiologic imaging of traumatic brain injury. Arch Phys Med Rehabil 2010;91:1661-66 